\title{
ZU LAND VON NEW YORK \\ AN DEN PANAMAKANAL \\ VOR 25 JAHREN UND HEUTE
}

PETER BURCKHARDT

Von New York über Saint-Louis, Laredo nach Mexico-City braucht der Zug in ununterbrochener Fahrt immer noch 5 Tage wie vor 25 Jahren. Die Züge sind heute nicht komfortabler oder etwa angenehmer als damals. Sie sind air-conditioned, haben 2 restaurants-cars, 2 saloons-cars mit Radio und der letzte Wagen besitzt eine Aussichtsplattform. In jedem Wagen hat es überdies Duschenräume. Fernsehen wie in den Transatlantikflugzeugen gibt es für diese lange Strecke jedoch nicht. Der Preis für die Eisenbahn $(180 \$)$ ist mehr als $35 \%$ höher als für die Flugzeugtouristenklasse ( $130 \$)$. Vor 25 Jahren war das Verhältnis gerade umgekehrt. Das Flugzeug braucht 5 Stunden.

Vor einem Vierteljahrhundert begann die Fahrt ab Mexico-City abenteuerlich zu werden. Darum schildere ich die Verkehrsverhältnisse hauptsächlich von dieser Stadt an südwärts. Kürzlich ist der Panamerican-Highway - einheimisch die «Carretera Interamericana» genannt, bis an den Panama-Kanal auch in der Regenzeit befahrbar fertiggestellt worden, und eine Straßenbrücke über den Kanal wurde eingeweiht. Jedoch führt noch keine Straße durch das östliche Panama nach Kolumbien und scheint auch in absehbarer Zeit nicht gebaut zu werden. Von der Grenze der USA bis Panama soll er gegen 3100 Meilen lang sein. Zweimal in der Woche, Montag und Donnerstag, gibt es einen Autocarkurs, der Guatemala-City 8 Uhr verläßt, um 16 Uhr in San Salvador eintrifft, am nächsten Morgen 4 Uhr weiterfährt und am gleichen Abend 19 Uhr Managua (Nicaragua) erreicht, dort um 4 Uhr des folgenden Tages aufbricht, um schon 13 Uhr in San José da Costa Rica anzukommen. Preis 30 \$. Bis Guatemala-City fährt auch heute noch die Eisenbahn, sie benötigt 40 Stunden bis an die Grenze und 12 Stunden bis zur Hauptstadt Guatemalas. Das Eisenbahnnetz ist nicht erweitert worden und die Reisezeiten haben sich kaum verbessert, weil heute mehr noch als damals für die größeren Distanzen die immer schneller und billiger werdenden Flugzeuge die Eisenbahn zunehmend verdrängen. Für einzelne und in der Regel lange Strecken wird dagegen nun das Reisen auf dem Panamerican-Highway mit dem eigenen Auto oder per Car der Bahn vorgezogen. 1938 wurden die Züge in Mexico-Chiapas manchmal von Räuberbanden überfallen und die Reisenden vollständig ausgeraubt. Nunmehr scheint diese Gefahr beseitigt. Über den Grenzfluß zwischen Guatemala und Mexico bestand noch keine Brücke. Mit Ruderbooten wurden die verschiedenen Arme des Flusses überwunden, wobei die Reisenden auf den Inseln dazwischen die Boote tragen helfen mußten. Der Panamerican-Highway war erst auf einigen unzusammenhängenden Teilstrecken ausgebaut. In Nicaragua dienten die Seen als Verkehrswege, in Honduras und Costa Rica die Flüsse. Weite Gebiete konnten nur auf Urwaldpfaden begangen oder beritten werden. Die Unterkünfte waren abseits der großen Städte schlecht. Wenn ich nicht hie und da bei Landsleuten aufgenommen worden wäre, hätte ich die Reise bald aufgegeben. Damals wurde allgemein das Meer als Reiseweg benützt. Von den Häfen in der heißen subtropischen Zone bestanden immer Straßen- oder Eisenbahnverbindungen zu den Städten in der höhergelegenen gemäßigten, angenehmen Zone. Wer sich also wie ich in den Kopf gesetzt hatte, auf dem Lande zu reisen, wurde als Schmuggler oder Revolutionär angesehen. Die Leibesvisitationen und peinlichst genaues Untersuchen des Gepäcks, die ich über mich ergehen lassen mußte, sowie langes Warten an den Grenzen waren unangenehm und zeitraubend. Neben dem Impfzeugnis, das auch heute noch verlangt wird, benötigte man damals in allen Staaten Zentralamerikas teure Visa mit 15 Photos und ärztliche Gesundheitszeugnisse. Wenn die Regierung unterdessen gewech- 


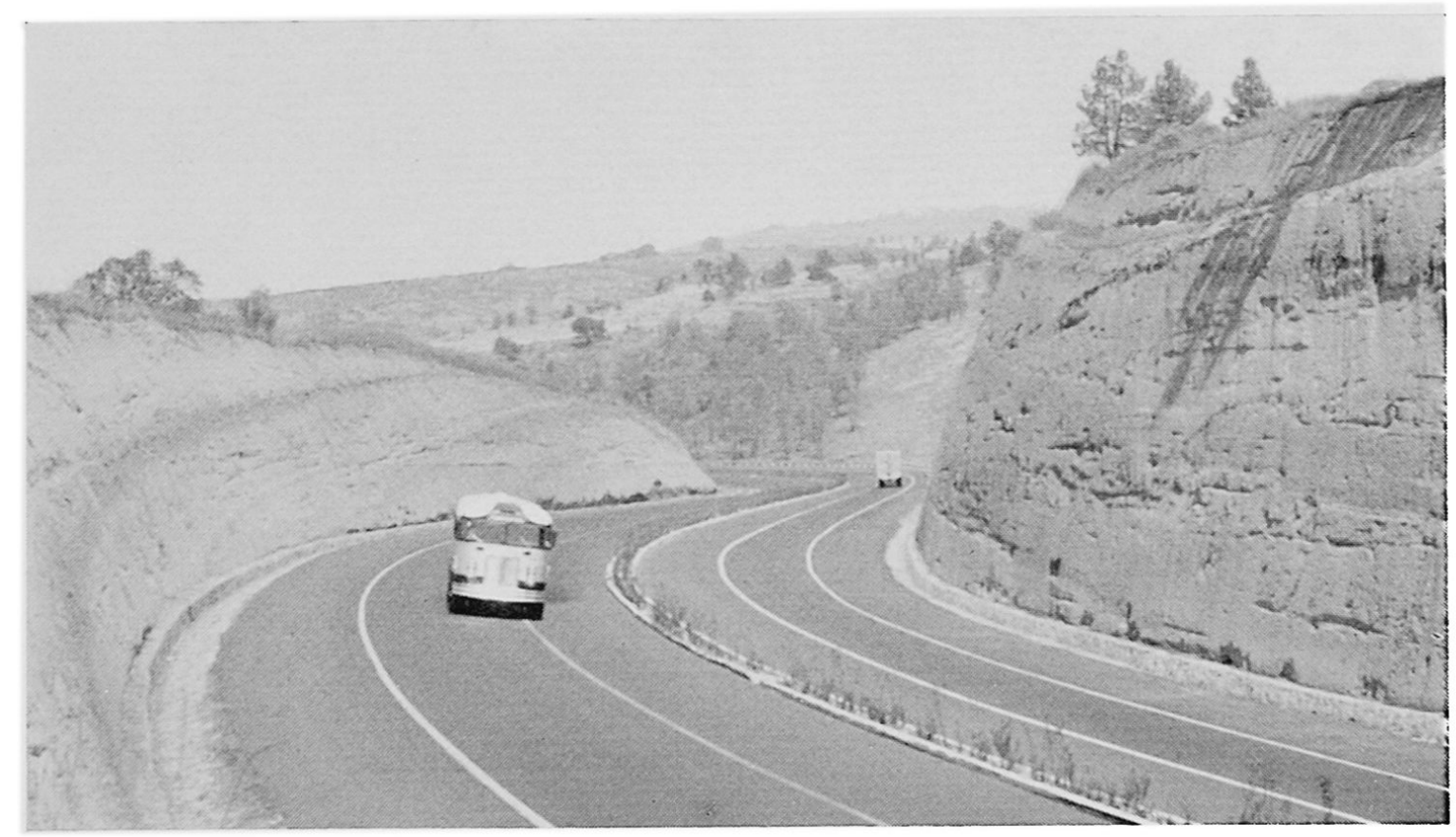

Abb. 1 Am Inter-American Highway zwischen Mexico City und Puebla. Nicht überall wie hier können die Fahrzeuge richtungsgetrennt verkehren.

(Photo Engineering News Record)

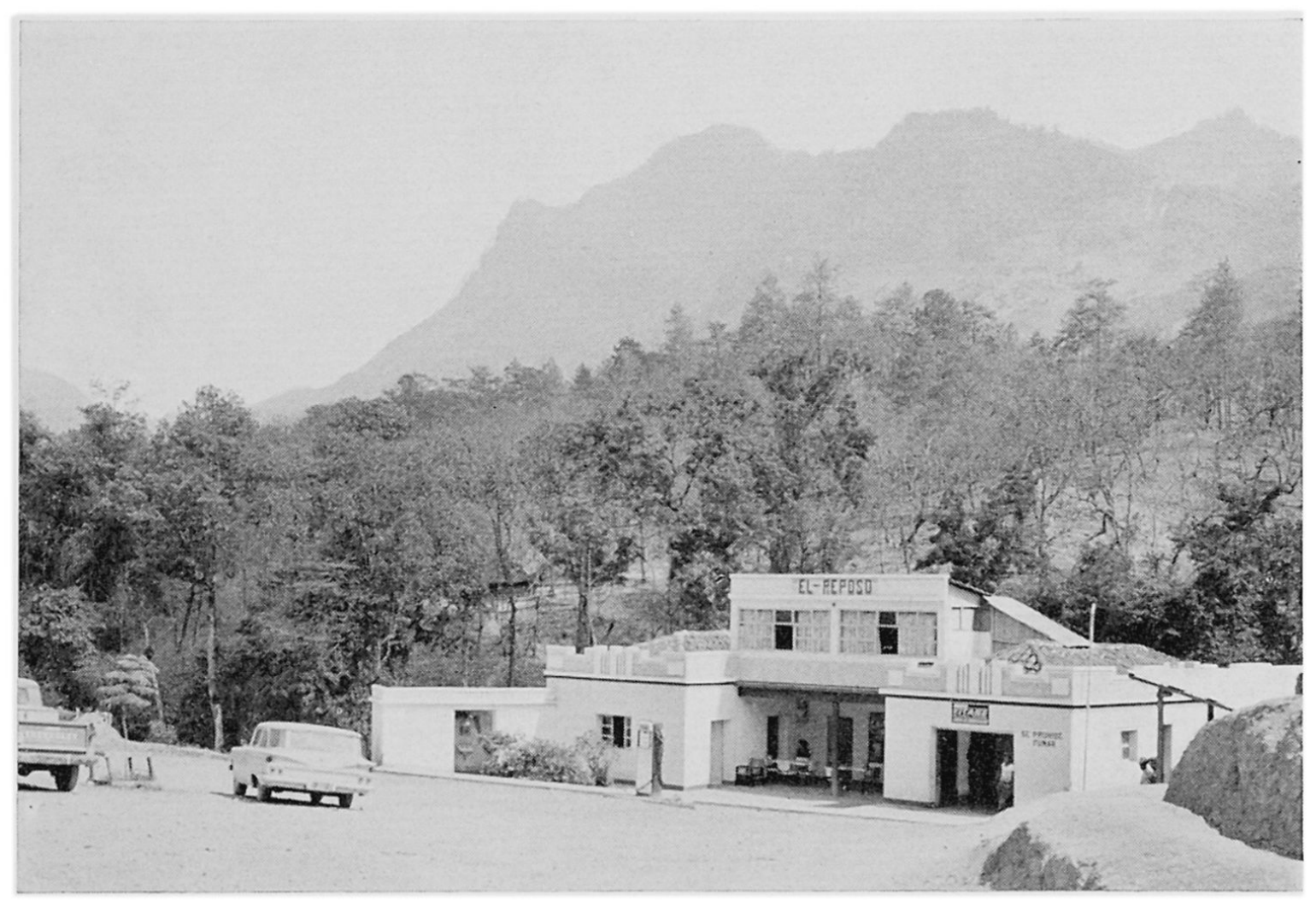

Abb. 2 Rasthaus und Service Station am Inter-American Highway südlich der mexikanischen Grenze in West-Guatemala.

(Photo Engineering News Record) 
selt hatte, wurden die lange erwarteten Einreisebewilligungen als ungültig erklärt und man mußte im Nachbarlande wiederum lange auf das neue Visum warten und auch neu bezahlen. In El Salvador hatte ich zudem an jedem Ort die Polizei aufzusuchen und brauchte auch noch ein Ausreisevisum. Heute sind für all diese Länder nur noch Touristenvermerke nötig, die man meist rasch gratis oder gegen geringe Gebühr erhält. El Salvador verlangt nur noch den gültigen $\mathrm{Pa}$. Heute ist der ganze Weg zu Land von New York an den Panama-Kanal in 14 Tagen gut zurückzulegen. Vor 25 Jahren brauchte ich dazu des Zehnfache.

Die Veränderungen der Landschaft erscheinen nicht so einschneidend, wie sich nach der explosionsartigen Zunahme der Bevölkerungszahl vermuten ließe. Diese wuchs von knapp 23 auf über 46 Millionen (etwa 1930-1960, Mexiko eingeschlossen). Dennoch blieb die mittlere Volksdichte unter 20 je Quadratkilometer, und nur El Salvador, die kleinste der zentralamerikanischen Republiken, die schon um 1930 gegen 80 Einwohner je Quadratkilomert gezählt hatte, vermochte diese Ziffer auf nahezu 120 zu steigern. Es kann deshalb nicht erstaunen, wenn die Anteile des landwirtschaftlich genutzten Landes verhältnismäßig niedrigen Ausmaßes waren und im Mittel nach wie vor weniger als $25 \%$, in El Salvador allein um $70 \%$, in Costa Rica, Guatemala, Panama und Britisch Honduras weniger als $20 \%$ ausmachen. Auch heute gilt deshalb die einprägsame Schilderung von $\mathrm{H}$. Boesch : «Ob man von Guatemala oder von Honduras nach El Salvador gelangt, für den Geographen ist der völlige Wechsel der Wirtschaftslandschaft am eindrucksvollsten. Schon im Anfluge beobachten wir, wie die bewaldeten Berglandschaften immer mehr zurücktreten und einer geschlossenen und dicht besiedelten Agrarlandschaft Platz machen. Überall sehen wir Häusergruppen und Dörfer, Straßen und Feldwege. Dorf folgi auf Dorf, voll von Kindern und Erwachsenen, die alle gegenüber dem ruhigen und gesetzten Guatemalteken lebhaftere Bewegungen und raschere Reaktionen zeigen. Neben den verschiedenen Anbaupflanzen für die Versorgung der örtlichen Märkte herrscht fast im ganzen Lande die Kaffeeproduktion in einer Ausschließlichkeit vor wie in keinem andern südamerikanischen Staate. Alle Anbaugebiete besitzen einen kurzen und billigen Zugang zu den Verladehäfen am Pazifischen Ozean». Noch immer dominiert der Kaffee mit zwei Dritteln des Ausfuhrwertes von 110-120 Millionen \$ den salvadorianischen Externhandel und damit wesentlich die Wirtschaft überhaupt. Aber inzwischen wurden doch auch andere $Z$ weige der Oekonomie gefördert; so stieg die Baumwollerzeugung um mehr als das Vierfache, die Zementproduktion um über das Dreifache und ähnlich ist bei vielen Industriezweigen ein verstärkter Ausbau zu beobachten, was nicht zuletzt auf die Vermehrung und qualitative Hebung der Schulen zurückzu führen ist, die auch den Anteil der Analphabeten um mehr als $15 \%$ senken ließen. Trotz den kaum weniger turbulenten politischen Verhältnissen als in andern lateinamerikanischen Ländern kann so El Salvador in verschiedener Hinsicht als eine kulturelle «Insel» im karibischen Raume gelten, die seine Bevölkerung denn auch nicht selten als «mittelamerikanische Schweiz» bezeichnet.

Um so mehr fallen die noch weiten «unberührten» oder von Menschen nur wenig belebten «Naturlandschaften» im übrigen Gebiet der «Landbrücke» auf. Einerseits ist dies darauf zurückzuführen, daß Zentralamerika mit Ausnahme von Guatemala, El Salvador und Mexiko schon früher, in der indianischen Zeit, verhältnismäßig wenig besiedelt war, andrerseits haben daran lokale und regionale Ungunstverhältnisse, die auch heute noch wirken, wesentlichen Anteil. Aber alle Länder zeigen in den letzten Jahrzehnten hohe Geburtenraten (über $45 \%$ ), so daß mit einem Andauern der Bevölkerungszahlen zu rechnen ist. Dies scheint paradox, wenn andrerseits festgestellt werden muß, daß Ruhr, Hakenwurmkrankheit und Malaria wie auch Gelbfieber noch die verbreitetsten Krankheiten sind und die Kindersterblichkeit nach wie vor erheblich ist. Mit Recht sagte vor kurzem H.-G. Gierloff-Emden: «Es gibt kaum eine Überlandfahrt, während der der Reisende nicht auf ein Kinderbegräbnis trifft. Nur wenige Familienmitglieder tragen eine kleine Holzkiste zum Friedhof, wo die kleinen weißen Holzkreuze 


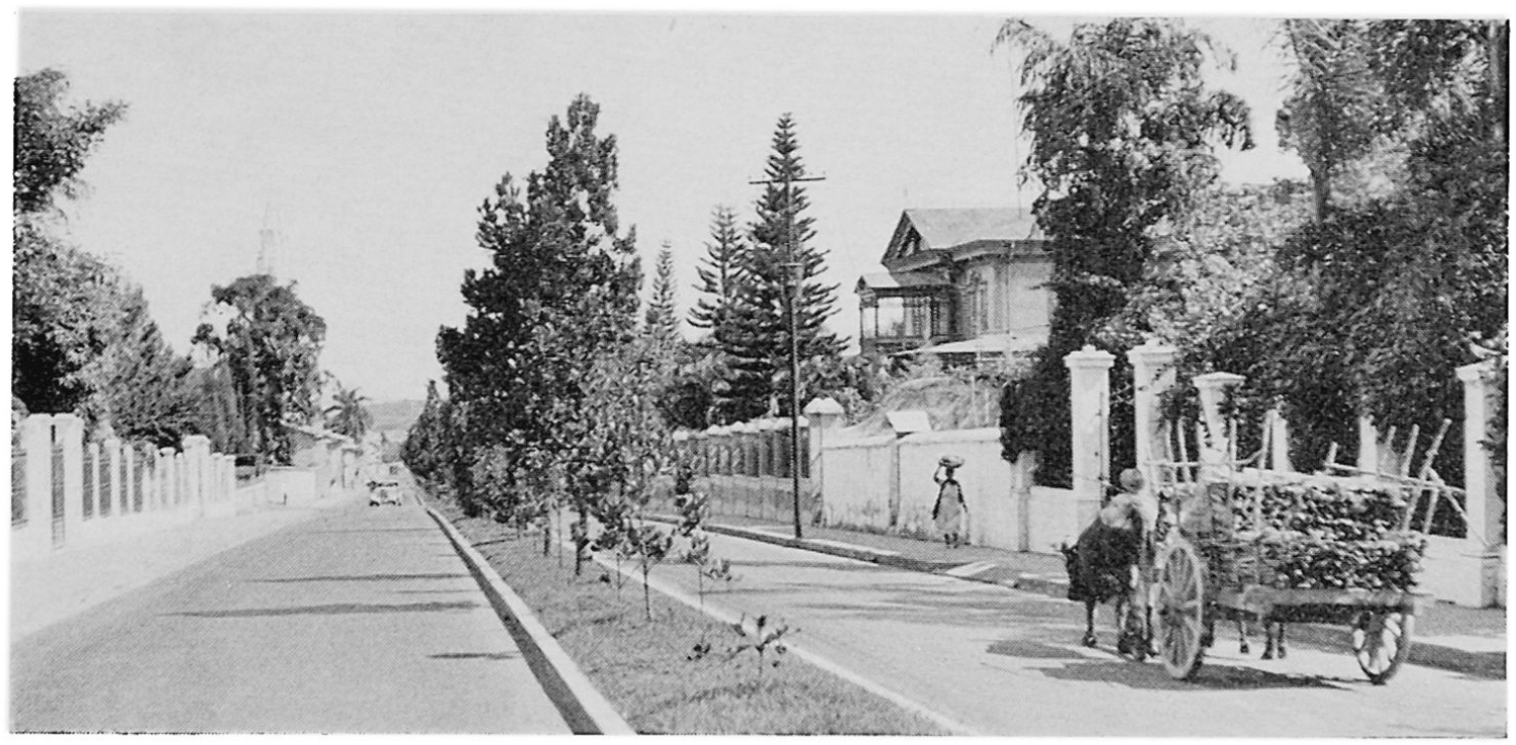

Abb. 3 Vorstadt von Guatemala. In diesen teilweise neuen Quartieren wird der Panamerican Highway, schon vor $25 \mathrm{Jahren}$ fertiggestellt, fast ebenso häufig von Ochsenkarren wie von Autos benützt.

(Photo P. Burckhardt)

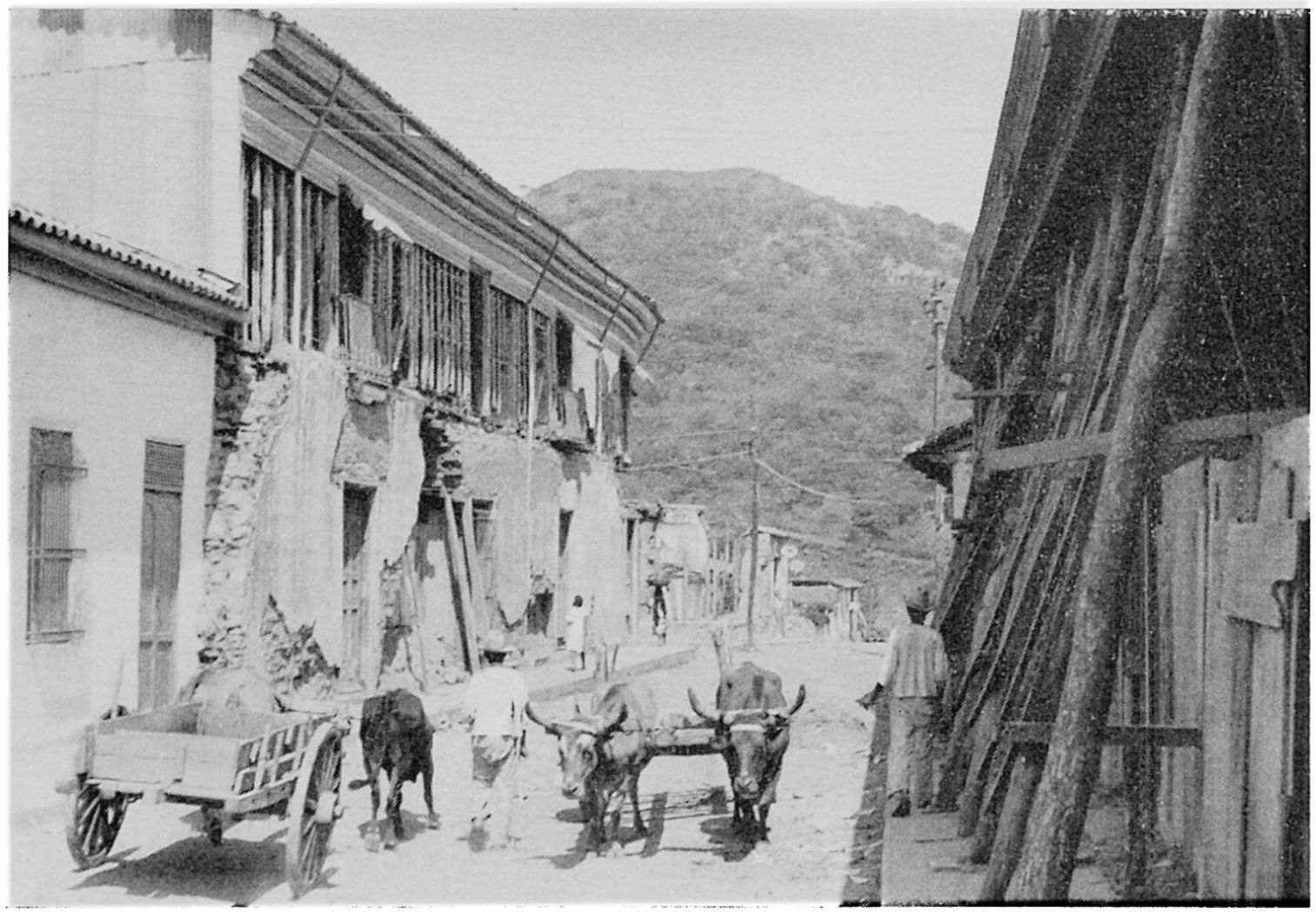

Abb. 4 Straße in San Miguel, El Salvador. Die Straße läßt deutlich Spuren eines Erdbebens erkennen, nach welchem die Bauten nur notdürftig geflickt wurden.

(Photo P. Burckhardt) 
stehen». Dieses Erlebnis belegt eine besonders bedrückende Konstante im sonst an Dynamismen reichen Entwicklungsbild Mittelamerikas. Sie wird aber dadurch unterstrichen, daß Hunderttausende von Landarbeitern nur über Hütten aus Stroh und Latten, primitive Herde, wenige Hängematten, Hemd, Hose, Strohhut, Sandalen und meist noch über eine Machete verfügen und bei einem Taglohn von 2-5 Franken (unter europäischen Preisverhältnissen!) naturgemäß einem höchst prekären Lebensstandard unterliegen, der die ebenso häufigen Mangelerscheinungen wie die primitiven Hygieneverhältnisse erklärt.

$\mathrm{Zu}$ den landschaftlichen «Konstanten» gehört in Mittelamerika auch die sich aus Klima und Relief ergebende vertikale Gliederung der Vegetation, die gleichermaßen in den «Stockwerken» der Natur wie den Wirtschaftslandschaften zum Ausdruck kommt. In der «tierra caliente», dem bis zu etwa 600 Meter Meereshöhe reichenden «heißen Land», gedeihen vor allem Bananen, Kakao, Zuckerrohr und Kokospalmen; die «tierra templada» oder gemäßigte Zone in 600 bis 1800 Metern ist Hauptanbaugebiet des Kaffees, in das - bis etwa $1600 \mathrm{~m}$ - jedoch auch Zuckerrohranpflanzungen hineingreifen. Über 1800 Metern erhebt sich die «tierra fria» oder kühle Region, wo Mais- und Weizenfelder mit «Nebel» und Kiefernwälder mit Schlingpflanzen, Orchideen und Moosen abwechseln und wo Nachtfröste sowohl den Aufenthalt als auch die Landwirtschaft erschweren und gefährden.

Diese Gebiete haben sich in den letzten 25 Jahren insofern geändert, als die krisenempfindlichen Monokulturen von Zuckerrohr in der Tierra caliente und von Kaffee in der gemäßigten und Hochlandzone auf der pazifischen Seite der Landbrücke mehr durch Baumwoll-, Tabak-, Reis-, Sisal- und Manilahanf sowie Weizenanbau ersetzt wurden. Auf der Atlantikseite beherrschen die Bananen noch immer das Feld. Die Technik der Betriebe hat Fortschritte gemacht, wenn auch nicht im gleichen Tempo wie in Nordamerika, nicht zuletzt deshalb, weil ein Überangebot an Arbeitskräften besteht, das allerdings durch die zunehmende Industrialisierung zurückzugehen scheint. Demgegenüber versorgen sich die Indianer der Hochländer von Guatemala, Honduras, Costa Rica und Panama nach wie vor mittels ihrer alten Landwirtschaft, mit Bohnen und Mais, und sie arbeiten auch ungern und selten in den Plantagen. Dort begegnet man deshalb im pazifischen Raum vornehmlich den Ladinos, im atlantischen den $\mathrm{Ne}$ gern und Mulatten. Die Indianer stehen Einmischungen von seiten der Andersrassigen mißtrauisch, ja aggressiv gegenüber, wenn auch im übrigen Rassendiskriminierung kaum Gewicht besitzt. Vor kurzem wurden staatliche Viehzähler in Guatemala verprügelt und fortgejagt.

Der Zug nach den Städten, die sich ständig vergrößert haben und weiterhin ausdehnen - nicht zuletzt vielfach auch verschönern - hält an wie überall in Amerika und der ganzen Welt. Doch liegt der Anteil der Stadtbevölkerung in Mittelamerika mit $44 \%$ (1960) tiefer als in Südamerika (46\%) und in Nordamerika (50-60\%). Das Gesicht der Städte ist weithin verändert worden; bis in die jüngste Zeit bestimmte es der spanische Kolonialstil, dem der schachbrettartige Grundriß, die zentrale «plaza», die rechtwinklig sich kreuzenden «calles» und «avenidas», die mehr oder weniger prunkvolle Kathedrale und das Stadthaus sowie andere öffentliche Gebäude das Gepräge verliehen. Die häufigen Erdbeben verhinderten mehrstöckige Bauten. Dies hat sich seit einiger Zeit geändert, die eingeschoßigen Wohnhäuser sind mehr und mehr aufgegeben worden, und an ihre Stelle traten 2- bis 3-stöckige Betonbauten, die den Beben besser widerstehen sollen als die frühern leichten Häuser. Noch stärker haben jedoch teils klassizistisch, teils moderne und ultramoderne Verwaltungsgebäude, Kinos, Geschäfte das Antlitz umgeformt, so daß die spanische Kolonialstadt, von wenigen Ausnahmen - etwa der «Ruinenstadt Antigua» - abgesehen, im Verschwinden begriffen ist.

Namentlich im städtischen Bereich Mittelamerikas wird so der Reisende zweifellos den Eindruck eines progressiven Aufbruches, einer ungemeinen Entwicklung erhalten, 

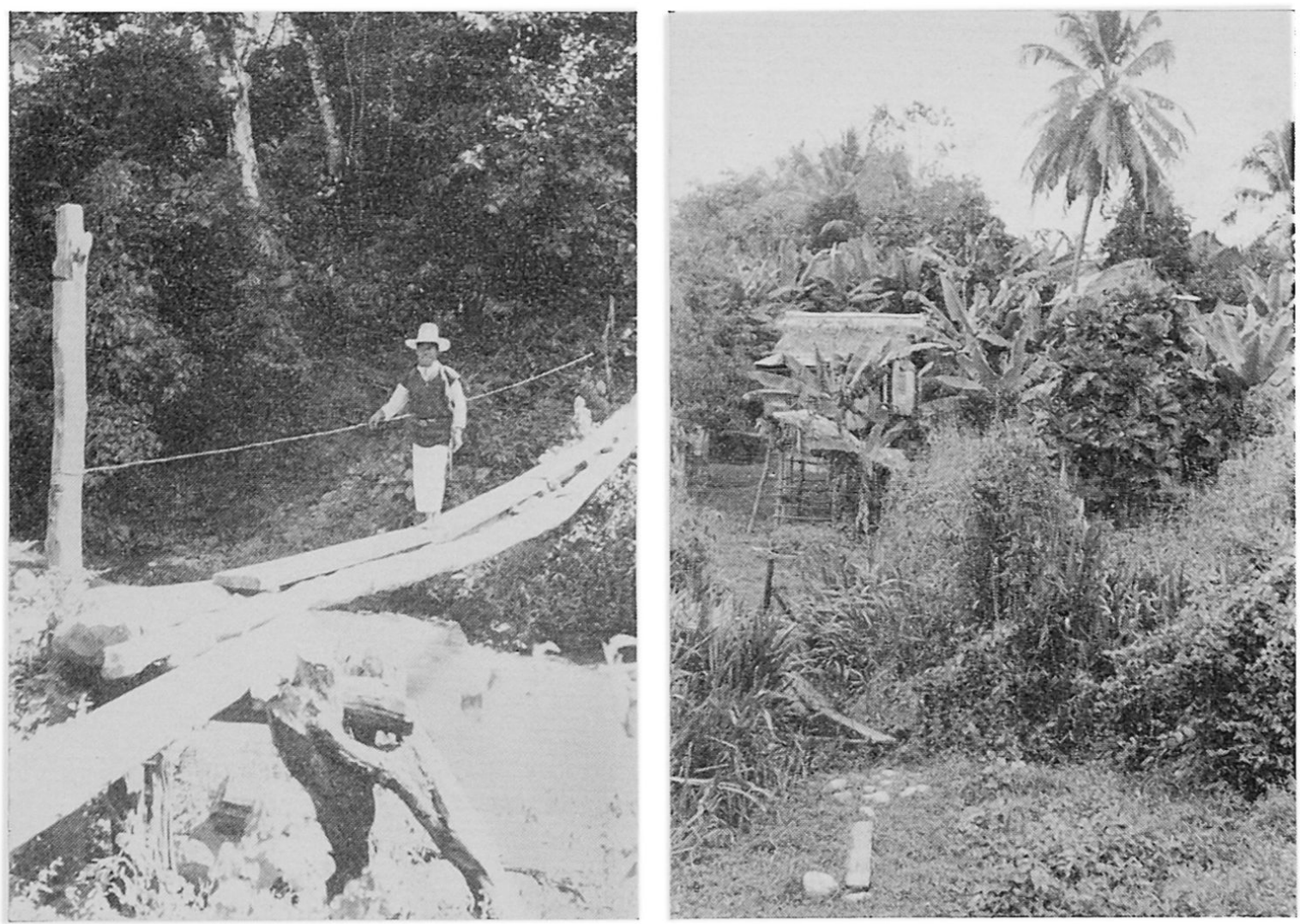

Abb. 5 «Brücke» über ein Urwaldflüßchen in Honduras. Abb. 6 Indianischer Pfahlbau im Wald der regenreichen atlantischen Küste Nicaraguas.

(Photo P. Burckhardt)

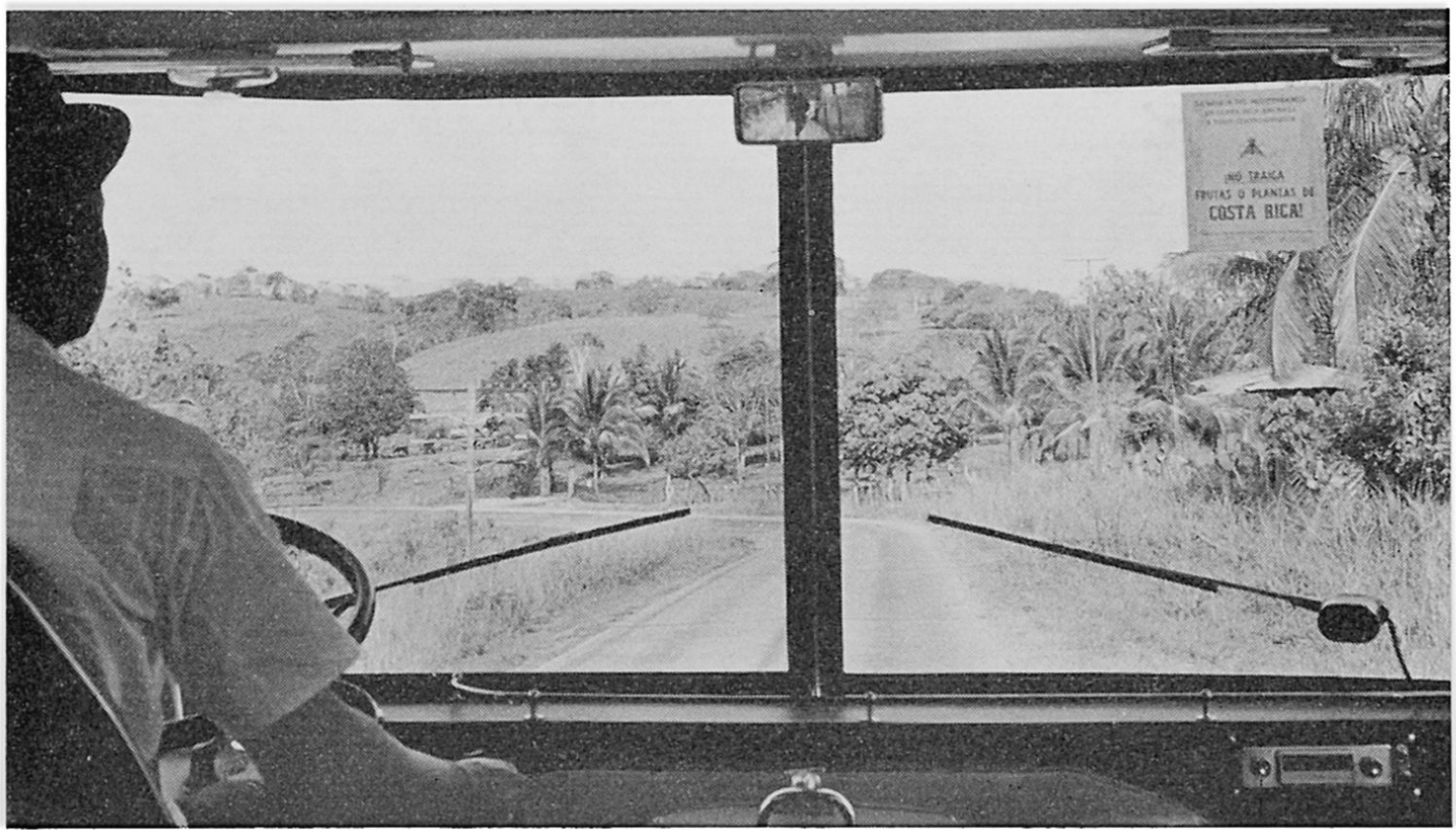

Abb. 7 Am Panamerican Highway in der Plantagenlandschaft Costa Ricas. Die Autobahn gleicht hier eher einer Landstraße.

(Photo Engineering News Record) 
dessen «Untergründe» freilich noch kaum erahnen lassen, ob seine Länder einer positiven oder negativen Transformation ihrer Kulturlandschaft entgegengehen werden.

\author{
DE NEW YORK A PANAMA PAR LA TERRE FERME IL Y A 25 ANS
}

Il y a 25 ans un voyage de New York à Panama par la terre ferme offrait beaucoup d'autres aspects qu'aujourd'hui. L'achèvement du «Panamerican Highway» a amélioré considérablement les voies de communication en Amérique Centrale. Après cette période nous constatons aussi par parties des changements importants dans le paysage culturel, ce qui est bien visible par le redoublement de la population (de 23 à 46 millions d'habitants). Nonobstant nous rencontrons encore toujours le long de cette route internationale les contrastes des régions densement peuplées, cultivées et d'un fort développement économique et des paysages d'une nature intouchée, bien qu'il y aît eu des progrès de différentes manières dans les états de l'Amérique Centrale.

\title{
DER BEGRIFF «TRANSHUMANZ» IM ENGLISCHEN SPRACHGEBIET
}

\author{
ROLF GUBLER
}

Im englischen Sprachgebiet erfreut sich der Begriff «Transhumanz» einer relativ großen Popularität. Dazu trägt allerdings die Tatsache viel bei, daß man darunter eine etwas andere Vorstellung hat als etwa im Bereich der romanischen Sprachen oder des Deutschen. So findet man z. B.im «German and English Glossary of Geographical Terms» (1 [siehe Anmerkungen]) : Transhumanz = Almwanderung. King (2) setzt Transhumanz mit Wanderung von Volksgruppen (Transhumance, or migration of population groups...) gleich und schreibt auch, daß perennierende Transhumanz (perennial transhumance) äquivalent zu Nomadismus sei. Gottmann (3) versteht unter Transhumanz in Frankreich die Wanderungen von Großvieh und Schafen, welche von Ställen oder Tieflandweiden aus auf die Alpweiden führen. In «Elements of Geography» (4) wird Transhumanz als eine besondere Form von «livestock ranching» charakterisiert, bei welcher die Sommerweiden gepachtet werden. Hoffmann (5) versteht unter Transhumanz Senntenwirtschaft oder Sennerei. Beinahe dieselbe unpräzise Definition wie Newbegin (6) (Transhumanz is the periodic and alternating displacement of flocks and herds between two regions of different climate) gibt Stamp (7), der unter Transhumanz Wanderungen zwischen Regionen verschiedener Klimate versteht. Ähnlich äußert sich auch James (8). Im Oxford Wörterbuch (9) versteht man noch allgemeiner einfach eine jahreszeitliche Wanderung von Vieh zu einer andern Region. Moore (10) präzisiert, daß bei Transhumanz die Herden von Hirten, manchmal auch von erheblichen Bevölkerungsteilen begleitet werden und daß diese sowohl in den Bergen als auch im Tal Dauersiedlungen bewohnen.

Auch in Spezialarbeiten wird unter Transhumanz oft eine Art von Viehhaltung verstanden, die der Alpwirtschaft oder Säterwirtschaft entspricht (11). Dazu gehört z. B. die Arbeit über Transhumanz in Nord Skye von MacSween (12). Ebenso häufig aber lassen sich aus den gegebenen Unterlagen nicht klar die Schlüsse ziehen, um was für eine Art der Viehhaltung es sich handelt, da über die wesentlichen Punkte hinweggegangen wird. Dies dürfte im Fall der Transhumanz in «Tasmanien» (13) zutreffen.

Stellt man die im englischen Sprachbereich üblichen Definitionen und Beschreibungen von Transhumanz den nicht-englischen (14) gegenüber, so ergeben sich ganz wesentliche Unterschiede. Im besondern versteht man unter Transhumanz im englischen Sprachbereich auch Wirtschaftsformen der Viehhaltung, die sonst ausdrücklich nicht 